\title{
WEAK SOLUTIONS WITH UNBOUNDED VARIATION
}

\author{
DONALD P. BALLOU ${ }^{1}$
}

\begin{abstract}
To solve a quasilinear system of hyperbolic partial differential equations with given initial data, the usual procedure is to approximate the initial data, solve the resulting problems, and show that the variation of the approximating solutions is uniformly bounded. A limiting process then can be used. This paper shows that, for simple systems, the variation of the solution need not be finite.
\end{abstract}

The usual procedure for solving the Cauchy problem for quasilinear hyperbolic systems is first to solve the problem obtained by approximating the initial data with a step function and then to use a limiting process. For this it is necessary that the variation of the approximating solution be uniformly bounded. ${ }^{2}$ This paper shows that for a simple system with bounded monotonic step data the weak solution will have infinite variation in the sense of Tonelli-Cesari.

The system considered is

where

$$
\frac{\partial v}{\partial t}-\frac{\partial f(u)}{\partial x}=0, \quad \frac{\partial u}{\partial t}-\frac{\partial v}{\partial x}=0
$$

$$
\begin{aligned}
f(u) & =\exp \left[-1 / u^{2}\right], & & u>0, \\
& =-\exp \left[-1 / u^{2}\right], & & u \leqq 0 .
\end{aligned}
$$

The initial data are $v^{0}(x) \equiv 0$ and $v^{0}(x)$ an increasing step function with an infinite number of jumps on a finite interval. The function $v^{0}(x)$ will be described more fully later on. Note that this system is not strictly hyperbolic and is not genuinely nonlinear.

It is first necessary to solve the Riemann problem with initial data

$$
\begin{aligned}
\left(u^{0}(x), v^{0}(x)\right) & =(0, \alpha), & & x \leqq 0, \\
& =(0, \beta), & & x>0,
\end{aligned}
$$

Received by the editors March 20, 1972 and, in revised form, May 15, 1972.

AMS (MOS) subject classifications (1970). Primary 35F25; Secondary 35B99.

Key words and phrases. Weak solutions, quasilinear hyperbolic systems, initial value problems, unbounded variation.

1 Partially supported by National Science Foundation Grant SD GU 3171.

${ }^{2}$ For example cf. Glimm and Lax [1], Johnson and Smoller [2]. 
with $\alpha<\beta$. This problem (in a more general form) has been handled by Leibovich [3]. We now describe those aspects of his work needed for this paper. Let $x_{1}<x_{2}$ and let $D_{*}\left(x_{1}, x_{2}\right)$ be the closed convex hull of the set $\left\{(x, y): x_{1} \leqq x \leqq x_{2}\right.$ and $\left.y \leqq f(x)\right\}$. Define a function $b_{*}=b_{*}\left(x ; x_{1}, x_{2}\right)$ on the interval $\left(x_{1}, x_{2}\right)$ by

$$
b_{*}\left(x ; x_{1}, x_{2}\right)=\max \left\{y:(x, y) \in D_{*}\left(x_{1}, x_{2}\right)\right\} .
$$

Let $u \geqq 0$ and consider the functions $F=F(u ; 0, \beta)$ and $B=B(u ; 0, \alpha)$ given by

$$
\begin{aligned}
& F(u ; 0, \beta)=\beta-\int_{0}^{u}\left[b_{*}^{\prime}(y ; 0, u)\right]^{1 / 2} d y, \\
& B(u ; 0, \alpha)=\alpha+\int_{0}^{u}\left[b_{*}^{\prime}(y ; 0, u)\right]^{1 / 2} d y .
\end{aligned}
$$

Let $I$ be any open interval with 0 as left-hand endpoint on which $f^{\prime \prime}>0$. Then $u \in I$ implies

$$
b_{*}^{\prime}(y ; 0, u)=\exp \left[-1 / u^{2}\right] / u, \quad 0 \leqq y \leqq u .
$$

Thus the $F$ and $B$ functions become

$$
\begin{aligned}
& F(u ; 0, \beta)=\beta-\left(u \exp \left[-1 / u^{2}\right]\right)^{1 / 2}, \\
& B(u ; 0, \alpha)=\alpha+\left(u \exp \left[-1 / u^{2}\right]\right)^{1 / 2} .
\end{aligned}
$$

If $\beta-\alpha$ is sufficiently small, then the curves defined by $v=F(u ; 0, \beta)$ and $v=B(u ; 0, \alpha)$ intersect at a point $(\bar{u}, \bar{v})$, where $\bar{u} \in I$. Note that $\bar{u}$ satisfies

$$
\bar{u} \exp \left[-1 / \bar{u}^{2}\right]=((\beta-\alpha) / 2)^{2} .
$$

Then the solution to the Riemann problem is given by

$$
\begin{aligned}
(u(t, x), v(t, x)) & =(0, \alpha), & x \leqq-\lambda t, \\
& =(\bar{u}, \bar{v}), & -\lambda t<x \leqq \lambda t, \\
& =(0, \beta), & x>\lambda t,
\end{aligned}
$$

where $\lambda=\left(\exp \left[-1 / \bar{u}^{2}\right] / \bar{u}\right)^{1 / 2}$. That this is the solution is easily verified by showing that the Rankine-Hugoniot condition holds along the curves of discontinuity and by proving that $x=-\lambda t$ is a backward shock while $x=\lambda t$ is a forward shock.

To define the Cauchy data $v^{0}(x)$ first let $\alpha_{0}>0$ be so small that the solution $a$ of $u \exp \left[-1 / u^{2}\right]=\alpha_{0}^{2} / 4$ belongs to the interval $I$. Next let $M$ be an integer so large that $\sum_{k=1}^{\infty}\left(1 /(k+1)^{M I}\right)<\alpha_{0}$. Define a sequence $\alpha_{0}, \alpha_{1}$, $\alpha_{2}, \cdots$ by

$$
\alpha_{k}=\alpha_{k-1}-1 /(k+1)^{M I}, \quad k=1,2,3, \cdots
$$


Note that $\bar{\alpha}=\lim _{k \rightarrow \infty} \alpha_{k}>0$. Let $u_{k}$ satisfy

$$
u_{k} \exp \left[-1 / u_{k}\right]=\left(\left(\alpha_{k}-\alpha_{k-1}\right) / 2\right)^{2}, \quad k=1,2,3, \cdots .
$$

Let $x_{1}$ be arbitrary and define a sequence $x_{1}, x_{2}, x_{3}, \cdots$ inductively by

$$
x_{k}=x_{k-1}-\lambda_{k}-\lambda_{k-1}, \quad k=2,3,4, \cdots,
$$

where $\lambda_{j}=-\left(\exp \left[-1 / u_{j}^{2}\right] / u_{j}\right)^{1 / 2}$. Note that $\bar{x}=\lim _{k \rightarrow \infty} x_{k}>-\infty$. This is because

$$
\sum_{k=0}^{\infty}\left(\frac{\exp \left[-1 / u_{k}^{2}\right]}{u_{k}}\right)^{1 / 2}<\infty
$$

a consequence of $u_{k} \rightarrow 0$ as $k \rightarrow \infty$.

Also observe that for $k=1,2,3, \cdots$ the backward shock originating at $x_{k}$ and the forward shock originating at $x_{k+1}$ interact at time $t=1$. Hence it is possible to define the weak solution $u(t, x)$ in the strip $0 \leqq t<1$. It is given by

$$
\begin{array}{rlrl}
u(t, x) & =\left(0, \alpha_{0}\right), & x & >x_{1}+\lambda_{1} t, \\
& =\left(0, \alpha_{k-1}\right), & x_{k}+\lambda_{k} t<x \leqq x_{k-1}-\lambda_{k-1} t, \\
= & \left(u_{k}, v_{k}\right), & x_{k}-\lambda_{k} t<x \leqq x+\lambda_{k} t, \\
=\left(0, \alpha_{k}\right), & x_{k+1}+\lambda_{k+1} t<x \leqq x_{k}-\lambda_{k} t, \\
=(0, \bar{\alpha}), & x \leqq \bar{x} .
\end{array}
$$

Here $v_{k}=\beta-\left(u_{k} \exp \left[-1 / u_{k}^{2}\right]\right)^{1 / 2}$. For each $t_{0}, 0<t_{0}<1$, the variation of $u\left(x, t_{0}\right)$ is given by $\sum_{k=1}^{\infty} 2 u_{k}$. Note that the variation takes place on the finite interval $\bar{x}-1 \leqq x \leqq x_{1}+\lambda_{1} t_{0}+1$. To see that $\sum_{k=1}^{\infty} 2 u_{k}=\infty$ we shall show that for all $k \geqq K, K$ sufficiently large, $u_{k} \geqq 1 /(k+1)$. To establish this, suppose otherwise. Then $u_{k}<1 /(k+1)$ holds for infinitely many $k$. But, for such $u_{k}$,

$$
\left(\left(\alpha_{k}-\alpha_{k-1}\right) / 2\right)^{2}=u_{k} \exp \left[-1 / u_{k}^{2}\right]<(1 /(k+1)) \exp \left[-(k+1)^{2}\right]
$$

and

$$
\left(\left(\alpha_{k}-\alpha_{k-1}\right) / 2\right)^{2}=1 / 4(k+1)^{2.1 I}
$$

together imply that

$$
1 / 4(k+1)^{2 M-1}<\exp \left[-(k+1)^{2}\right]
$$

which is impossible for $k$ sufficiently large.

The above results are summarized in the following theorem.

THEOREM. Consider the initial calue problem

$$
\frac{\partial v}{\partial t}-\frac{\partial f(u)}{\partial x}=0, \quad \frac{\partial u}{\partial t}-\frac{\partial v}{\partial x}=0
$$


with initial data $u^{0}(x) \equiv 0$ and $v^{0}(x)$ bounded and monotone increasing. Then for certain $v^{0}(x)$ there are functions $f \in C^{\infty}$ for which the weak solution has unbounded variation in the sense of Tonelli-Cesari on certain compact subsets of the half plane $t \geqq 0$.

\section{REFERENCES}

1. J. Glimm and P. D. Lax, Decay of solutions of systems of nonlinear hyperbolic conservation laws; Mem. Amer. Math. Soc. No. 101 (1970). MR 42 \#676.

2. J. L. Johnson and J. A. Smoller, Global solutions for an extended class of hyperbolic systems of conservation laws, Arch. Rational Mech. Anal. 32 (1969), 169-189. MR 38 \#4822.

3. L. Leibovich, Solutions of the Riemann problem for hyperbolic systems of quasilinear equations without convexity conditions, J. Math. Anal. Appl. (to appear).

Department of Mathematics, State University of New York, Albany, New YORK 12203 